\title{
GROUP ACTIONS AND EQUIVARIANT LIPSCHITZ ANALYSIS
}

\author{
MEL ROTHENBERG AND SHMUEL WEINBERGER
}

The idea that one could prove by analytic methods the topological invariance of characteristic classes was suggested in an influential lecture by Singer. The concrete realization of this, in the case of rational Pontrjagin classes, was the result of works by Sullivan, who showed that topological manifolds have essentially unique Lipschitz structures, and Teleman, who showed that Lipschitz manifolds are the type of object for which one can analyze the signature operator. (A refinement of the same method proves the $K O[1 / 2]$ orientability of topological bundles.)

Much of this can be done for manifolds with group actions.

The main implication is that for certain sorts of topological group actions (including all smooth and PL actions) one can construct an "equivariant signature operator" whose class in equivariant $K$-homology is a topological invariant. This class satisfies the $G$-signature theorem. The topological method has implications for various precise classification problems (e.g. semifree topological actions on the sphere), but here we shall present only the consequences of these Lipschitzian ideas:

(A) Topologically locally linear $G$-manifolds for odd-order $G$ have $K O_{G}[1 / 2]$ orientations (Madsen-Rothenberg).

For all finite $G$ there are Lipschitz structures, but the signature class is a zero divisor for even-order groups. The same method produces, upon application of the localization theorem, "Atiyah-Singer" classes for some actions with nonmanifold fixed set (Weinberger, extending earlier joint work with Cappell).

(B) For odd-order groups, topological and linear conjugacy of linear representations are equivalent (Hsiang-Pardon, Madsen-Rothenberg).

This is a consequence of (A). Alternatively one can return to the original argument of Atiyah-Bott-Milnor for semifree representations.

A stronger result is true: the Atiyah-Bott local numbers associated to representations agree for elements that have discrete fixed sets in topologically equivalent representations. This distinguishes many pairs of representations of even-order groups.

(C) $R \operatorname{Top}(G)=R \operatorname{Lip}(G)$ for all finite $G$, where $R$ Cat is the Grothendieck group of representations up to Cat conjugacy (Weinberger, after inverting 2).

This implies that there is no analytic definition of Reidemeister torsion that works well in the Lipschitz setting. The reason is that, following

Received by the editors December 20, 1986.

1980 Mathematics Subject Classification (1985 Revision). Primary 57S17; Secondary 57R99, 58A99.

The authors are supported by NSF grant DMS-860-2980 and the second author is an NSF Presidential Young Investigator. 
de Rham, Rothenberg has given a torsion theoretic proof that PL conjugacy is equivalent to linear conjugacy. Since $R$ Top $\neq R O$ by the famous examples of Cappell and Shaneson, torsion cannot be made to function as in invariant in the Lipschitz category. In the smooth case Ray and Singer have given an analytic description of Reidemeister torsion for free actions, as verified by Cheeger and Müller, and Rothenberg has observed that these works can be extended to cover the nonfree case.

(D) For $G$ actions that have $\pi_{1}(M) \rightarrow \pi_{1}(M / G)$ split injective a form of the equivariant Novikov higher signature conjecture holds provided $\pi_{1}(M)$ is a discrete subgroup of a connected Lie group.

This follows from identifying the class of the Lipschitz signature operator with an equivariant algebraic Poincaré complex. (The details are in Rosenberg-Weinberger, following ideas of Kaminker-Miller.) By far the deepest part is proving the result for $G$, the trivial group, and $M$ smooth; this is due to Kasparov.

(E) The key idea in constructing equivariant Lipschitz structures is the following lemma (and its analogue for Lipschitz homeomorphisms):

LEMMA. For every transfer-invariant Lipschitz group action on $T^{k} \times S^{n}$ there is such an extension to $T^{k} \times D^{n+1}$.

This is proven geometrically by making use of the torus covers that unwrap all directions equally. One continually makes use of the fact that Lipschitz constants can be related to the $L^{\infty}$ norms of partial derivatives, so that all coverings of actions have a uniform bound on their Lipschitz constants (for their induced metrics) and that uniform rescaling does not change Lipschitz constants.

One makes extensive use of Quinn's theory of ends of maps to show that after crossing an action with a torus, one is in a situation that can be handled by repeated use of the lemma. The hypothesis of the lemma is assumed inductively, and one starts the induction with Sullivan's construction.

The method is perhaps best explained by first concentrating on the twostrata case. First suppose that there is a single fixed point. If the action had an invariant sphere near the fixed point, one can use Sullivan's theorem to make the action on it Lipschitz, and extend that "Lipschitzinization" to the complement of the neighborhood. The action on the neighborhood can be replaced by the cone on the action on the boundary (engulfing shows that this is topologically equivalent to what we started with). Unfortunately, such a sphere does not always exist. However, after crossing with $S^{1}$ an invariant $S^{1} \times S^{n-1}$ does exist. Furthermore, one can deduce the existence of a transferinvariant one, and then apply the lemma. If the fixed set is more than a point, one tries to do the same thing, by triangulating the fixed set (at least locally, and then applying Sullivan's ideas to get around this difficulty using small Lipschitz handle decompositions) and trying to get a block structure on this neighborhood. Again there are obstructions, but after crossing with a torus they go away. (Here the dimension has to go up because you have to solve $k$ end problems to get the projection of a mapping cylinder structure "transverse" to a codimension $k$ submanifold, and you need one $S^{\mathbf{1}}$ to get 
the mapping cylinder structure.) Then one inducts over the skeleta of the triangulation.

In general, one inducts on the number of strata. This stably constructs equivariant Lipschitz structures (of a rather special kind) whenever the fixed sets are all locally flat submanifolds. (These are the weakly stratified actions of Quinn.) Stable uniqueness follows from a relative form of existence.

(F) One can construct, in the locally linear case, unique equivariant Lipschitz locally linear structures, even without crossing with tori. This makes use of the stability of $\pi_{0}$ of $G$-homeomorphism groups, to show that all homeomorphisms can be approximated by ones that can be made Lipschitz using the lemma. This is necessary to deal with zero handles. The other handles are dealt with using work of Siebenmann-Sullivan on Lipschitz homeomorphisms of polyhedra.

\section{REFERENCES}

M. Atiyah, R. Bott and A. Lefschetz, Fixed-point theorem for elliptic complexes, II: Applications, Ann. of Math. (2) 88 (1968), 546-604. 355 .

S. Cappell and J. Shaneson, Nonlinear similarity, Ann. of Math. (2) 113 (1981), 315-

J. Cheeger, Analytic torsion and the heat equation, Ann. of Math. (2) 109 (1979), 259 321.

G. de Rham, Reidemeister's torsion invariant and rotations of $S^{n}$, Differential Analysis, Oxford, Oxford Univ. Press, 1964, pp. 27-36.

W. C. Hsiang and W. Pardon, When are topologically equivalent orthogonal representations linearly equivalent? Invent. Math. 68 (1982), 275-316.

J. Kaminker and J. Miller, Homotopy invariance of the analytic index of signature operators over $C^{*}$-algebras, J. Operator Theory 14 (1985), 113-127.

G. Kasparov, K-theory, group $C^{*}$-algebras and higher signatures (conspectus), preprint, Chernogolovka, USSR, 1981.

I. Madsen and M. Rothenberg, On the classification of $G$-spheres. I-II, 1985, Aarhus preprints.

W. Müller, Analytic torsion and R-torsion of Riemannian manifolds, Adv. in Math. 28 (1978), 233-305.

F. Quinn, Ends of maps. II, Invent. Math. 68 (1982), 353-424. , Weakly stratified spaces, 1985, preprint.

D. Ray and I. Singer, R-torsion and the Laplacian on Riemannian manifolds, Adv. in Math. 7 (1971), 145-210. print.

J. Rosenberg and S. Weinberger, Higher G-indices and their applications, 1986, pre-

M. Rothenberg, Torsion invariants and finite transformation groups, Proc. Sympos. Pure Math., vol. 32, Amer. Math. Soc., Providence, R.I., 1978.

L. Siebenmann and D. Sullivan, On complexes that are Lipschitz manifolds, Geometric Topology (Proc. Georgia Topology Conf., Athens, Ga., 1977), Academic Press, New York, 1979, pp. 503-525.

I. Singer, Future extensions of index theory and elliptic operators, Prospects in Math., Ann. of Math. Studies, no. 70, 1971, pp. 171-185.

M. Steinberger and J. West, Controlled finiteness is the obstruction to equivariant handle decomposition, preprint.

D. Sullivan, Hyperbolic geometry and homeomorphisms, Geometric Topology (Proc. Georgia Topology Conf., Athens, Ga., 1977), Academic Press, New York, pp. 543-555.

$\mathrm{N}$. Teleman, The index of the signature operator on topological manifolds, Inst. Hautes Études Sci. Publ. Math. 58 (1983), 39-78. 
The index theorem for topological methods, Acta Math. 153 (1984), 117-152.

S. Weinberger, The Lipschitz rationality of linear representations, Comm. Pure Appl. Math. (to appear).

, Atiyah-Singer classes for singular actions, in preparation.

Department of Mathematics, University of Chicago, Chicago, Illinois 60637 